\title{
Antigen Processing
}

National Cancer Institute

\section{Source}

National Cancer Institute. Antigen Processing. NCI Thesaurus. Code C17662.

The process by which foreign molecules are taken up by antigen presenting cells,

proteolytically cleaved, and displayed on the cell surface in complex with MHC molecules. 